\title{
AN ASSESSMENT OF THE RECENT DEVELOPMENT OF MEMS OSCILLATORS AS COMPARED WITH CRYSTAL OSCILLATORS
}

\author{
C.S. LAM \\ TXC Corporation, Ping Cheng City, Taoyuan County, Taiwan
}

\begin{abstract}
Due to its high Q and temperature stable properties, for many years, quartz crystal based oscillators are important clock sources in consumer, commercial, industrial, and military digital sub-modules and modules. The demand for quartz crystal resonators and oscillators continues to rise. The unique fabrication and encapsulation requirements though render quartz crystal resonators and oscillators difficult or close to impossible to be integrated onto the mature silicon based IC platforms. The recent technical breakthroughs of MEMS (Micro Electro Mechanical Systems) based resonators and oscillators seem to re-ignite the interest in displacing/replacing the quartz crystal technology and to open up again the prospect in clock source integration.

This paper discusses and assesses, from the viewpoint of a quartz crystal manufacturer, such development and its possible impact on the quartz crystal industry which also experiences major progresses in miniaturization, performance enhancement, cost reduction, etc., in the past few years. This paper is not to discredit the MEMS oscillator efforts but to help the quartz crystal manufacturers to understand more about the efforts and advise them what they need to prepare for.
\end{abstract}

Keywords: MEMS, quartz, resonator, crystal oscillator

\section{Introduction}

Due to its high Q and temperature stable properties, for many years, quartz crystal based oscillators are important clock sources in consumer, commercial, industrial, and military digital sub-modules and modules. $^{[1]}$ The demand for quartz crystal resonators and oscillators continues to rise. The unique fabrication and encapsulation requirements though render quartz crystal resonators and oscillators difficult or close to impossible to be integrated onto the mature silicon based IC platforms.

For a typical handset, four different piezoelectric components are needed- RF SAW filter $(\sim 900 \mathrm{MHz}$ to 2 $\mathrm{GHz}$ using $\mathrm{LiTaO}_{3}$ or $\mathrm{LiNbO}_{3}$ ), IF SAW filter ( $\sim 50$ to $400 \mathrm{MHz}$ using mostly quartz), and TCXO (temperature-compensated crystal oscillator, $\sim 10$ to 30 $\mathrm{MHz}$ and quartz-based) in the RF section; and tuning fork (32.768 KHz and quartz-based) for standby clocking in the baseband section (Fig. 1).

Quite a few years ago the successful development of direct conversion technology in transceiver chipsets rendered IF SAW filter obsolete in may GSM handsets (as in Fig. 1). Some GSM transceiver chipsets now have the digitally-compensated crystal oscillator (DCXO) circuit of the TCXO integrated on-chip. ${ }^{[2]}$ However, a quartz crystal is still needed off-chip (Fig. 2). As the handset market continues to grow, developing Micro Electro Mechanical Systems (MEMS) components as switches, filters, resonators/oscillators, etc. in the RF section of handsets seems to be the logical route of the final solution- integration (Fig. 3).
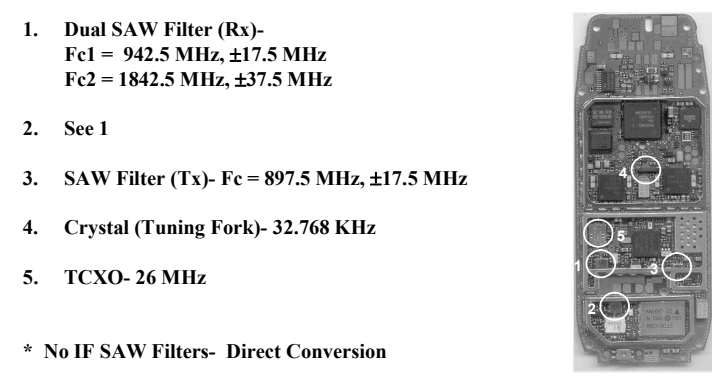

Fig. 1 Piezoelectric Components in a Typical Dual Band GSM Handset (GSM900 and DCS1800)

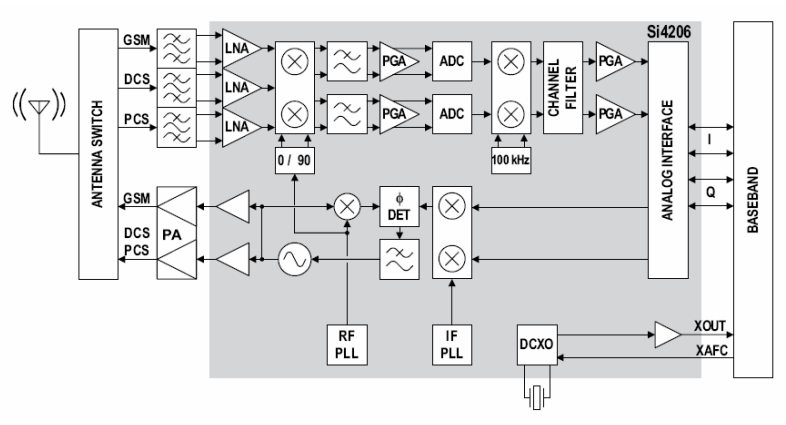

Fig. 2 GSM Transceiver Chipset with On-chip DCXO ${ }^{[2]}$

About 15 to 25 years ago, thin film resonators (TFRs) were vigorously discussed and researched as they could 
possibly be integrated onto the mature silicon based IC platforms. $^{[3]}$ After that it came 5 years of a quiet period. In 1994, HP research personnel presented the first report on film bulk acoustic resonator (FBAR) ${ }^{[4]}$ and in 2001 Agilent announced the production of FBAR-based duplexers. FBAR (also coined BAWR nowadays by some), with its small size, silicon etching process and sacrificial layer removal step, can be considered as a MEMS component. As of now, FBAR-based duplexers and filters have successfully replaced a small portion of the rooted RF SAW duplexer/filter market but they still have to be individually packaged- like the RF SAW devices. Discrete RF SAW filter (and/or FBAR), TCXO (or quartz crystal) and quartz tuning fork are still the key off-chip piezoelectric components in handsets. FBAR integration and the full scale MEMS integration as in Fig. 3 are not likely to happen in the foreseeable future. ${ }^{[5]}$

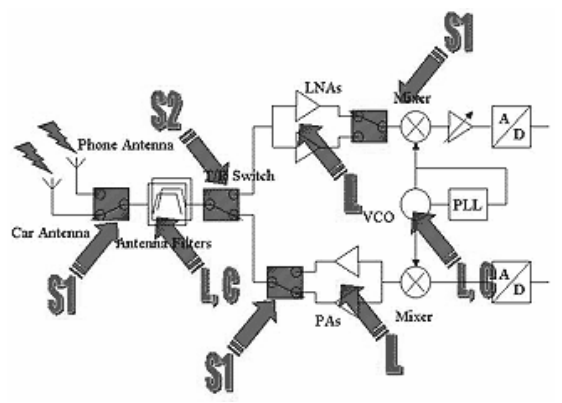

Fig. 3 Applications of MEMS in RF Section of Handsets ${ }^{[5]}$

The recent technical breakthroughs of MEMS based resonators and oscillators seem to re-ignite the interest in displacing/replacing the quartz crystal technology and to open up again the prospect in clock source integration. This paper discusses and assesses, from the viewpoint of a quartz crystal manufacturer, such development and its possible impact on the quartz crystal industry which also experiences major progresses in miniaturization, performance enhancement, cost reduction, etc., in the past few years. This paper is not to discredit the MEMS oscillator efforts but to help the quartz crystal manufacturers to understand more about the efforts and advise them what they need to prepare for.

\section{Silicon Solutions}

Silicon clock oscillators are CMOS integrated circuits without using ceramic resonators, quartz crystals or other external components for frequency determination. The author commented in $2003^{[6]}$ on such solution based on the Maxim's EconOscillator/Divider and Micro Oscillator's MOI-2000 Oscillator (Fig. 4). ${ }^{[7]}$ One can easily note that the frequency stability of these oscillators is 5,000 to
$15,000 \mathrm{ppm}$ and so their applications are somewhat limited.

Linear Technology also offers a family of silicon oscillators with stability ranging from 2,500 to 15,000 ppm. ${ }^{[8]}$ Recently, Mobius Microsystems announced the offering of the "All-silicon Copernicus Clock with 2,500 ppm frequency stability over process, voltage, and temperature.",[9]

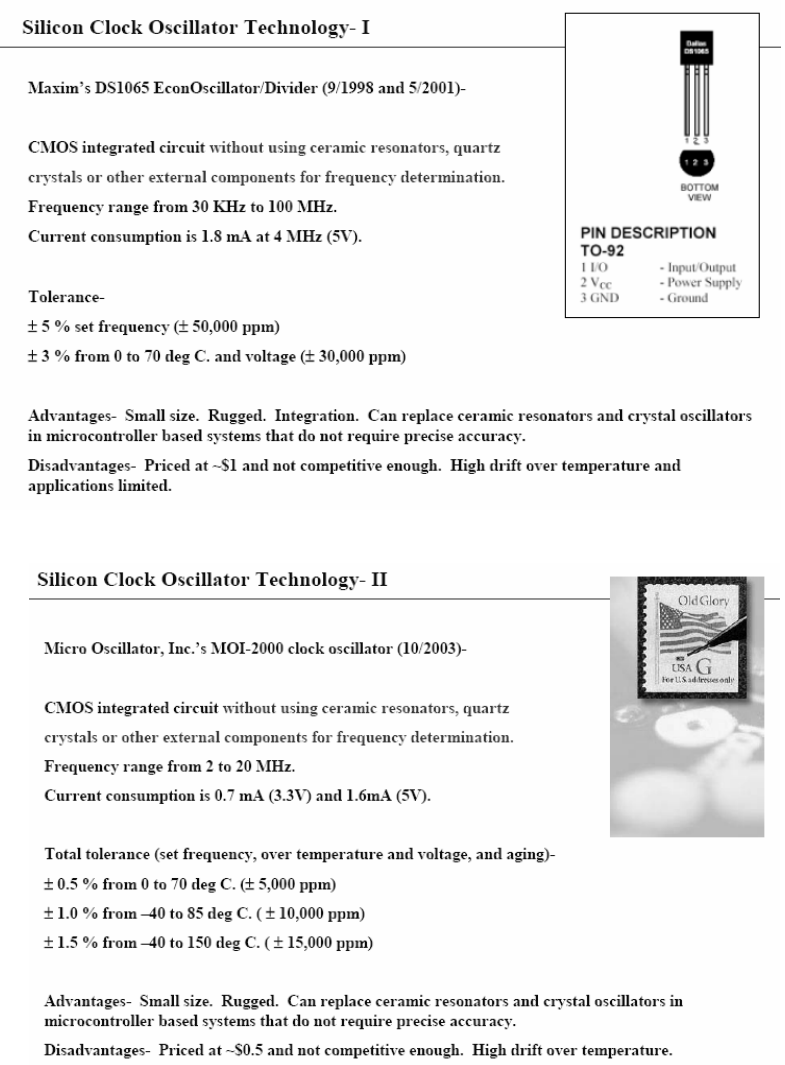

Fig. 4 Silicon Clock Oscillator Technology, I- Maxim's EconOscillator/Divider and II- Micro Oscillator's MOI-2000 ${ }^{[7]}$

Silicon clock oscillators can be integrated onto other silicon based IC platforms. One big advantage is their ruggedness (suitable for high shock and vibration applications) as there is no discrete mechanical resonant element. Their biggest disadvantage is clearly their poor frequency stability.

\section{Quartz Solutions}

It is almost 80 years since the applications of quartz crystals in telecommunication equipment first appeared. ${ }^{[1]}$ Due to its high $Q$, unchallenged frequency-temperature stability, low cost, technical maturity, and widely commercial availability, quartz 
crystal continues to be the choice, and sometimes the only choice, in providing stable frequency sources in the ever expanding digital world.

\begin{tabular}{|l|l|}
\hline BAW-based $(<M H z \sim 200 \mathrm{MHz})$ & SAW-based $(<50 \mathrm{MHz} \sim 3 \mathrm{GHz})$ \\
$\begin{array}{l}\text { Passives- } \\
\text { Tuning Fork and Resonator }\end{array}$ & SAWR \\
MCF & SAWF \\
Oscillators- & CSO \\
CXO & \\
PCXO & VCSO \\
VCXO (Voltage-Controlled) & TCSO? \\
TCXO (Temp. Compensated) & OCSO? \\
OCXO (Oven-Controlled) & \\
Timing Modules- & Clock Data Recovery (CDR) \\
Clock Data Recovery (CDR) & Clock Smoother (CS) \\
Clock Smoother (CS) & Frequency Translator (FX) \\
Frequency Translator (FX) & STM \\
STM & $\ldots$. \\
\hline$\cdots$ & \\
\hline
\end{tabular}

Table 1 BAW (Crystal)- and SAW-based Passives, Oscillators and Timing Modules

Powered by the fierce growth in wired and wireless equipment, quartz crystal (Bulk Acoustic Wave based = BAW-based) and its high frequency version SAW device (Surface Acoustic Wave based) are being used widely ranging from a simple passive resonator to a complex synchronous timing module (STM) as show in Table 1.

Though unthinkable a few years ago, AT-cut quartz crystal (resonator) as small as $2.5 \times 2 \mathrm{~mm}^{2}$ is now being shipped in volume (Fig. 5). ${ }^{[10]}$ Applications which need even smaller quartz crystals $\left(2 \times 1.6\right.$ and $\left.1.6 \times 1 \mathrm{~mm}^{2}\right)$ are expected to appear and quartz crystal manufacturers are getting ready for them. Smaller than $5 \times 3.2 \mathrm{~mm}^{2}$ quartz crystals need to be sealed in vacuum to retain impedance integrity. Low $\mathrm{MHz}$ small size quartz crystal blanks also need to be beveled (contoured) to sustain efficient energy trapping.

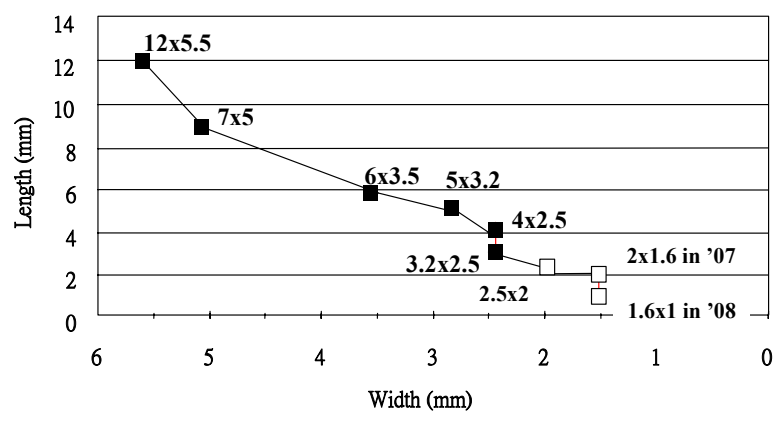

Fig. 5 Development of Quartz Crystals

As for the crystal oscillators, delivery of $2.5 \times 2 \mathrm{~mm}^{2}$ CMOS fixed frequency clock crystal oscillators (XOs/CXOs) is ramping up (Fig. 6) and quartz crystal manufacturers are preparing the yet smaller $2 \times 1.6 \mathrm{~mm}^{2}$ version. ${ }^{[10]}$ Oscillator IC suppliers also continue to shrink the IC sizes and for the $2.5 \times 2 \mathrm{~mm}^{2} \mathrm{CXO}$ one can still use wirebonding instead of flipchipping (Fig. 7).

CMOS programmable clock crystal oscillators (PCXOs) are widely available in $7 \times 5,5 \times 3.2$ and $3.2 \times 2 \mathrm{~mm}^{2}$ sizes. ${ }^{[1]}$ These oscillators have on-chip phase lock loops (PLLs) with at least two dividers to allow programming to almost any frequency within a working frequency range (e.g. 2 to $200 \mathrm{MHz}$ ) based on a single low frequency quartz crystal (e.g. $25 \mathrm{MHz}$ ). These oscillators are attractive to designers as they can be quickly programmed to the frequencies desired for development purpose but they suffer from higher jitter which is inherent to all PLL-based oscillators. CXOs with differential LVPECL/LVDS output are also available in $14 \times 9$ and $7 \times 5 \mathrm{~mm}^{2}$ platforms. Recently, one supplier began to offer programmable LVPECL CXO. ${ }^{[12]}$ The frequency stability of the above quartz crystal, $\mathrm{CXO}$ and PCXO is usually specified at less than 100, 50, 25 and $10 \mathrm{ppm}$ dependent on the temperature range of operation- significantly better than the all silicon solutions.

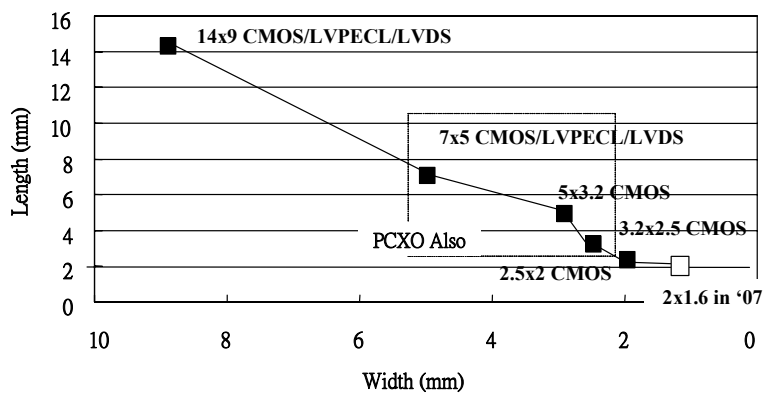

Fig. 6 Development of Crystal Oscillators
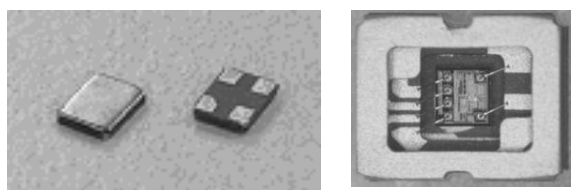

Fig. $72.5 \times 2 \mathrm{~mm}^{2}$ CMOS Crystal Oscillators ${ }^{[10]}$

To provide even better frequency stability, temperature-compensated crystal oscillator (TCXO) is available. AT-cut quartz crystal's frequency-temperature stability is cubic in nature. The oscillator IC can have added voltage-frequency pulling function to analogously or digitally compensate the cubic frequency-temperature as depicted in Fig. 8. For nowadays handset applications, a frequency stability of better than $2.5 \mathrm{ppm}$ TCXO is usually needed to provide the accurate reference clock of frequency synthesizing within the RF transceiver IC. For GPS equipment, TCXO of less than 1 or $0.5 \mathrm{ppm}$ is needed. In 2004, the smallest TCXO available was $3.2 \times 2.5 \mathrm{~mm}^{2}$ (Fig. 9). ${ }^{[13]}$ 
Now it is $2.5 \times 2 \mathrm{~mm}^{2}$.

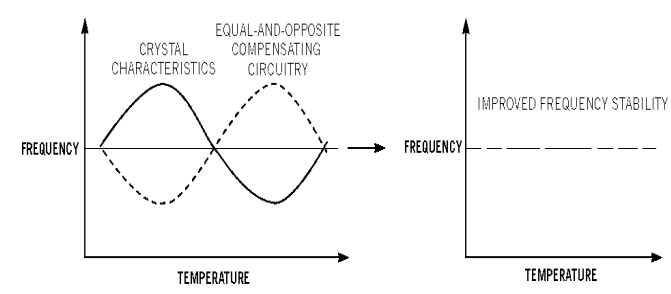

Fig. 8 Temperature Compensation of Quartz Crystal for TCXO Applications.

Overall, in the past few years, quartz crystal manufacturers have done the what once thought was impossible in shrinking the sizes of quartz crystal, CXO, PCXO and TCXO to where we are and most important of all, without sacrifying performance and cost. ${ }^{[14,15]}$ Claims and threats to displace/replace quartz crystal devices come and go. More and more quartz crystal devices are shipped yearly and the average selling price continues to drop.

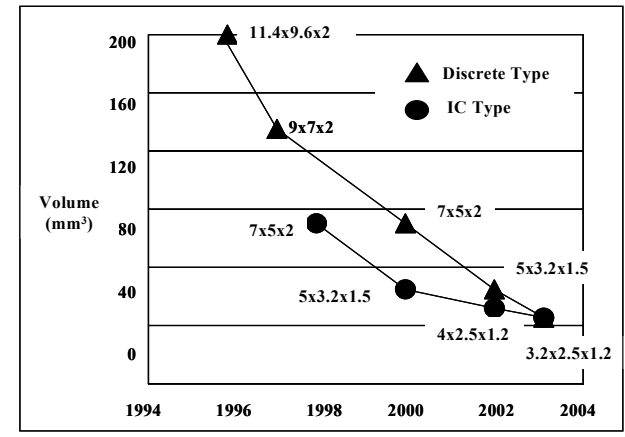

Fig. 9 Development of TCXO for Handset Applications till 2004

\section{MEMS Solutions}

Suggesting to use MEMS techniques to build the resonant elements for oscillator applications is not something new. The MEMS resonant elements are usually micromachined on silicon and they come in the shapes of combs, beams, discs, etc. For quartz crystal resonators, the vibration is based on piezoelectric excitation. For MEMS resonant element, it's based on electrostatic dynamics with "transducer gap" typical less than $1 \mu \mathrm{m}$ (Fig. 10). ${ }^{[16,17]}$ Similar to the silicon clocks discussed in Section 2, MEMS resonators are rugged as they are small and integration is possible.

In 1998, Sandia National Laboratories announced "Microscopic machines may replace quartz crystals, Pollen-grain-sized parts soon in watches, TVs, computers."[18] Based on the earlier MEMS works funded by DARPA ${ }^{[19]}$, Discera was established in 2001 with the mission "to become a global leader in CMOS MEMS resonator technology and to offer a broad portfolio of patented PureSilicon ${ }^{\mathrm{TM}}$ resonators whose breakthrough technology is proven reliable and used to create the industry's most advanced and economical Frequency Control and RF Circuits ..... Discera is the trend setter in today's multi-billion dollar timing industry, displacing quartz crystal solutions with systems-on-a-chip alternatives." ${ }^{,[20]}$
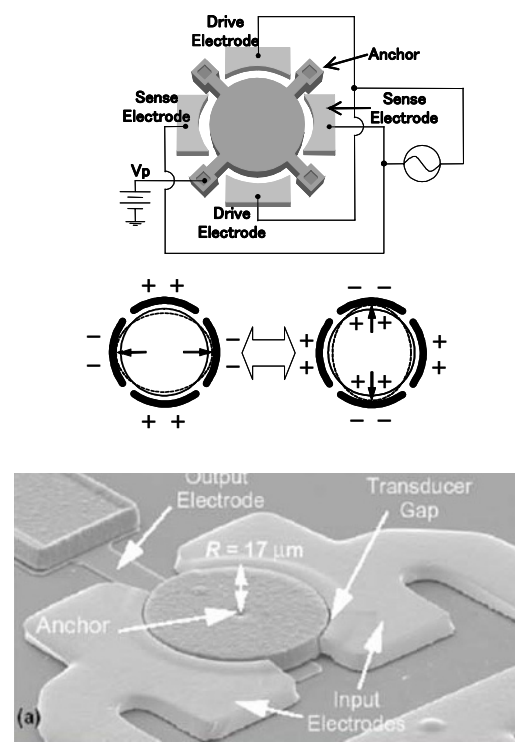

Fig. 10 Electrostatic Dynamics of Disc-Like MEMS Resonator ${ }^{[16,17]}$

In 2003, Discera offered the first MEMS oscillator MRO-100 $\left(3 \times 3 \mathrm{~mm}^{2}\right)$ in $19.2 \mathrm{MHz}$ for multiband wireless transceiver application (Fig. 11). The resonant element was a $30 \mu \mathrm{m} \times 8 \mathrm{~m}^{2}$ MEMS beam.

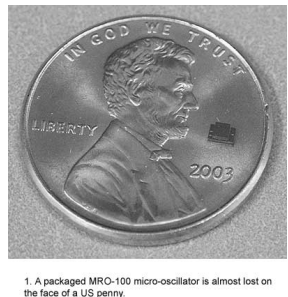

Fig. $113 \times 3 \mathrm{~mm}^{2}$ MEMS Oscillator ${ }^{[20]}$

In 2003, Forman ${ }^{[21]}$ commented "the challenge for Discera is in convincing customers to abandon an entrenched, known technology (quartz crystal technology) in favor of something new- convincing them to go with the devil they don't know." In 2004, Discera also demonstrated the first integrated $1.6 \mathrm{GHz}$ tunable oscillator, likely for the VCO (usually discrete LC-based) of the local oscillator in wireless transceiver applications. 
As of today, no detailed specifications of the above two oscillators are available in the public domain and it's difficult to assess their acceptability in the commercial market.

Based on the IP licensed through Bosch, SiTime was established couple of years ago with the mission to become "a fabless integrated circuit company developing silicon timing, clock, and RF chips, which incorporate MEMS timing reference devices inside standard silicon electronic chips, eliminating the need for quartz crystals." ${ }^{[22]}$ The first products introduced were the SiT8002 series programmable oscillators and the SiT1 series fixed frequency oscillators (likley programmed from the SiT8002 series). The CMOS oscillators are available in $7 \times 5,5 \times 3.2,3.2$ and $2.5 \times 2 \mathrm{~mm}^{2}$ (Fig. 12)clearly targeting at the pin-to-pin compatible quartz crystal oscillator market (Fig. 6).

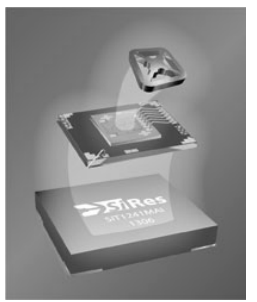

Fig. 12 SiTime's MEMS Oscillator ${ }^{[22]}$

The key success by SiTime over previous MEMS efforts was that "the clean and high vacuum sealing technology developed was able to minimize contamination and to support low aging of the MEMS resonator."[23,24] The SiT8002 and SiT1 series oscillators started out with a packaged 4-beam MEMS resonator (Fig. 13) wirebonded onto a leadframe-supported oscillator IC, and the whole assembly was then plastic-molded.
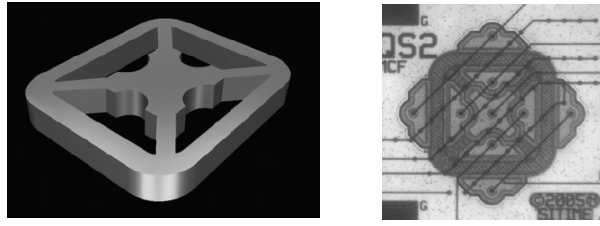

Fig. 13 MEMS Resonator based on 4-Beam Structure ${ }^{[22]}$

Little information was available on the MEMS resonator until SiTime announced the offering of it as a SiT0100 part (Fig. 14). The MEMS resonator operates at 5.1 $\mathrm{MHz}$ with 6-pad connection which is different from the familiar 2-pad connection of a quartz crystal resonator. The know-how in driving the MEMS resonator, Q and resistance values of the resonator, programming methodology of the oscillator, etc. are only available under a non-disclosure fees-associated agreement. No commercial available oscllator ICs are known to be available yet in pairing with this MEMS resonator.

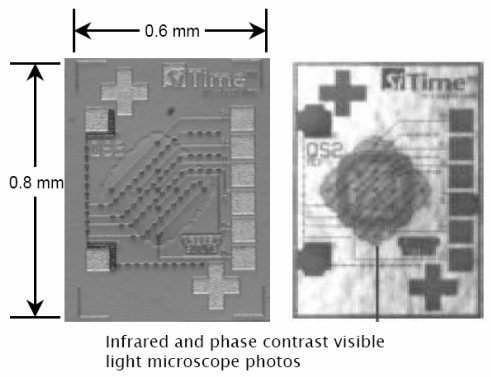

Fig. 14 Packaged 0.8x0.6x0.15mm3 MEMS Resonators ${ }^{[22]}$

Based on the public information available, one can say the SiT8002 series MEMS oscillators have a $5.1 \mathrm{MHz}$ MEMS resonator inside and the oscillator output frequency is programmed (PLL'ed) from this MEMS resonator- similar to the PCXOs mentioned in Section 3. It is of interest to mention the series number SiT8002 is remarkably similar to that of the SG-8002 series PCXO from a major crystal oscillator manufacturer. ${ }^{[11]}$ Recalling silicon-based MEMS resonator in general exhibits an inherent $\sim-30 \mathrm{ppm} /{ }^{\circ} \mathrm{C}$ frequency stability. To achieve the frequency stability claimed by the MEMS oscillators (say 50 to $100 \mathrm{ppm}$ ), temperature compensation is needed- similar to the TCXOs mentioned in Section 3 except likely a simpler linear compensation is performed. In summary, it current MEMS oscillators need to be-

programmed to frequency (like $P C X O$ ) and programmed to compensate frequency stability (like TCXO).

A PCXO needs to go through the following steps after encapsulation- tested, programmed, and tested (all done at room temperature). A TCXO needs to go through the following elaborate and costly steps after encapsulation- frequency-temperature test over the temperature range of operation, compensation, and frequency-temperature verification test over the temperature range of operation. Since the silicon-based MEMS resonator exhibits a linear frequency-temperature stability, compensation over the entire temperature of operation might not be needed to achieve a few tens of ppm stability. To get better stability, compensation based on frequencies measured at several temperatures close to the room temperature might be needed. Based on the information available so far, several questions with regard to the cuurent generation off MEMS oscillators can be posted-

Higher current drain than regular $C X O$ to power the PLL circuits? 
Poorer jitter performance as it's inherent to PLL-based oscillators?

Digital compensation often creates frequency perturbations within small temperature increments. Would that present problems for many applications?

Plastic molding is not hermetic- limitation in applications?

Programming and compensation can be costly?

As for integration, Johnson ${ }^{[25]}$ commented "Though Discera's MEMS is cast into a CMOS chip, neither its nor SiTime's MEMS chips hold circuitry. Today, adding MEMS to a CMOS chip with circuitry would risk turning the circuitry to soup by virtue of the high temperatures needed to sculpt MEMS structures from silicon. Consequently, like quartz crystals, MEMS resonators today require a separate CMOS chip, containing a feedback path to maintain oscillation, a PLL to stabilize frequency and other conditioning circuitry to optimize performance. In the case of quartz crystals, the CMOS chip is wired to the crystal inside the package. By contrast, the MEMS resonator, as a planar CMOS chip itself, can be directly bonded to the chip holding the circuitry." As said in the introduction, FBAR integration is not likely to happen in the foreseeable future. The author believes it applies to MEMS oscillators also.

\section{Quartz Plus MEMS Solutions}

As said in the introuction, "FBAR, with its small size, silicon etching process and sacrificial layer removal step, can be considered as a MEMS component.”

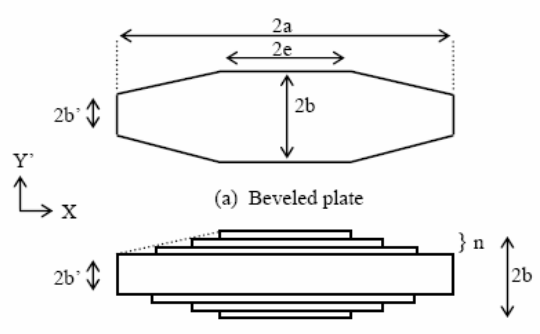

(b) Multi-stepped bi-mesa plate

Fig. 15 (a) Beveled Quartz Crystal and (b) Bi-Mesa Quartz Crysta ${ }^{[26]}$

Nowadays many quartz crystal components are miniaturized and some sorts of MEMS processing steps are involved- like the double-side photolithography, etch through, $\mathrm{Au}$ etch protection, etc. of miniature quartz tuning forks; etching steps of bi-mesa type quartz crystals (Fig. 15) ${ }^{[26]}$, Lamé mode quartz crystal ${ }^{[27]}$; etc. A major crystal oscillator manufacturer already coined the term "QMEMS" in recognizing the importance of linking the quartz and MEMS technologies for the next generations of quartz crystal devices. ${ }^{[28]}$ Recently both Discera and SiTime announced their alliances with two individual quartz crystal manufacturers. ${ }^{[29,30]}$ It seems instead of pushing pure MEMS-based solutions, some MEMS companies consider the best route is to link up with the quartz crystal manufacturers to identify markets which can best use both the quartz and MEMS techniques.

\section{Discussions}

This paper discusses and assesses, from the viewpoint of a quartz crystal manufacturer, the performances of silicon clocks and MEMS oscillators as compared with quartz crystal oscillators. This paper is not to discredit the MEMS oscillator efforts but to help the quartz crystal manufacturers to understand more about these products. The author believes MEMS oscillator technology has yet many issues (technical, cost, acceptance, etc.) to overcome in order to compete with the rooted quartz crystal oscillator technology. ${ }^{[31]}$ The quartz crystal industry, though experiences major progresses in miniaturization, performance enhancement, cost reduction, etc. in the past few years, shall have to continue to retain its competitiveness through further

\section{miniaturization,}

cost reduction,

performance improvement,

ease of use improvement,

reliability improvement,

development of new applications,

investigation of Quartz + MEMS techniques, etc.

\section{References}

1. "A History of the Quartz Crystal Industry in the USA, ” Virgil E. Bottom. Proc. 1981 Annual Frequency Control Symposium, pp. 3-12, http://www.ieee-uffc.org/fc history/bottom.html.

2. AERO ${ }^{\mathrm{TM}} I+$ Tranceiver for GSM and GPRS Wireless Communications, Silicon Laboratories, http://www2.silabs.com/public/documents/tpub doc /dsheet/Wireless/Aero_RF Transceivers/en/aeroI+.p df.

3. "Sputtered AlN Films for Bulk-Acoustic-Wave Devices, ” J.S. Wang and K.M. Lakin, Proc. 1981 Ultrasonics Symposium, pp. 502-505.

4. "Micromachined Thin Film Bulk Acoustic Wave Resonators, ” R. Ruby and P. Merchant, Proc. 1994 
IEEE Int'l Frequency Control Symposium, pp. 135-138.

5. "MEMS in CMOS- 21st Century RF and Microwave Applications, " Patrick Albert, John Costello and Patrick Salomon, RFdesign, July 2000.

6. "Applications of Frequency Control Products for Telecommunication \& Handset and Their Development Trends, " IT Market Conference, Dec. 19, 2003, Beijing, http://www.txc.com.tw/download/TECH PAPER/20 03-ITMarket-1-English.pdf.

7. "Microcontroller Clock-Crystal, Resonator, $R C$ Oscillator, or Silicon Oscillator?”' Maxim's Application Note 2154 http://www.maxim-ic.com/appnotes.cfm/appnote_nu mber/2154 and "MOI-2000 CMOS Clock Oscillator," Micro Oscillator, http://www.micro-oscillator.com/MOI2000_Data\%2 0Sheet4-04.pdf.

8. Linear Technology Silicon Oscillators, http://www.linear.com/pc/viewCategory.do?navId= $\mathrm{H} 0, \mathrm{C} 1, \mathrm{C} 1010, \mathrm{C} 1096$.

9. "Mobius Microsystems Introduces the All-silicon Copernicus Clock,” May 10, 2005, http://www.us.design-reuse.com/news/news10402.ht $\underline{\mathrm{ml}}$.

10. TXC Corporation's 2006 Products Roadmap.

11. Epson-Toyocom SG-8002 Series Programmable Crystal Oscillators, http://www.epsontoyocom.co.jp/english/product/OS C/programmable.html.

12. "Versatile OTP Oscillator Delivers Differential LVPECL for Data Designers with Deadlines," C-MAC MicroTechnology, September 1, 2005, http://news.thomasnet.com/fullstory/467366.

13. "Development of Miniaturized Analog and Digital Temperature Compensated Crystal Oscillators, " C.S. Lam and C.W. Chiang, Proc. Second Symposium on Acoustic Devices for Future Mobile Communication Systems, March 3 5, 2004, Chiba, Japan.

14. "Why oscillators are the best frequency sources for wireless designs, ” Roger Burns, RFdesign, August 1, 2003,

http://rfdesign.com/mag/radio_why_oscillators best /index.html.

15. "It's Crystal Clear: Quartz Still Resonates with Designers, ” Jon Titus, ECN, October 1, 2006, http://www.ecnmag.com/article/CA6380016.html?fi lename=ECN20061001 ec6ocs100 .xml.

16. "Current Activities of Japanese Quartz Industries," Masanobu Okazaki, Proc. 100th Meeting of the No. 150th Committee on Acoustic Wave Devices, Japanese Society for the Promotion of Science, Dec. 4 5, 2006, Taipei, Taiwan.

17. "Design and Fabrication Procedure for High $Q$ MEMS Resonators, "Wan-Thai Hsu, Microwave
Journal, February 1, 2004, pp. 60-74.

18. "Microscopic machines may replace quartz crystals, Pollen-grain-sized parts soon in watches, TVs, computers, " Sandia National Laboratories, News Release, July 7, 1998, http://www.sandia.gov/media/memquar.htm.

19. "Transceiver Front-end Architectures using High-Q Micromechanical Resonators, ” C. T.-C. Nguyen, Proc. IEEE European MIDAS Workshop, University of Surrey, United Kingdom, July 17-18, 2000.

20. Discera, http://www.discera.com.

21. "Discera's dilemma: Making enough noise to merit mobile's attention," David Forman, Small Times, June 23, 2003,

http://www.smalltimes.com/document_display.cfm? document id=6261

22. Sitime, http://www.sitime.com.

23. "Silicon MEMS Oscillators to Replace Quartz Crystals," Aaron Partridge and John McDonald, SiTime Corporation's 2006 Promotion Materials.

24. "A New Paradigm in Time: Silicon MEMS Resonators vs. Quartz Crystals," Thomson Dialog NewsEdge, May 1, 2006, http://www.tmcnet.com/usubmit/2006/05/01/162753 $\underline{4 . h t m}$.

25. "MEMS Eyes Quartz Crystal Apps," R. Colin Johnson, EE Times, October 23, 2006 http://www.embedded.com/rss/showArticle.jhtml?ar ticleID $=193401321 \&$ cid=RSSfeed embedded news

26. "Decoupling Effect of Multi-Stepped Bi-Mesa AT-Cut Quartz Resonator, " S. Goka et al., Proc. 2003 IEEE International Frequency Control Symposium, pp. 694-697.

27. "Quasi-Lamé-Mode Quartz-Crystal Resonators," Yasutaka Shimizu, et al., Japan. J. Appl. Phys. Vol.39 (2000), Part 1, No. 5B, 30 May 2000, pp. 3010-3013.

28. "What is QMEMS?" Epson-Toyocom, http://www.epsontoyocom.co.jp/english/info/2006/q mems.html.

29. "Vectron and Discera to Drive New Era of MEMS Timing Devices, ” November 14, 2006, http://www.discera.com/03b zp111406.shtml.

30. "SiTime and Micro Crystal Enter MEMS Oscillator Joint Development Agreement," November 13, 2006, http://www.sitime.com/news/releases/micro111306. $\underline{\mathrm{htm}}$.

31. "MEMS oscillators, has their time come?" Jérémie Bouchaud and Bernardo Knoblich, Wicht Technologie Consulting, http://www.memsinvestorjournal.com/2006/10/mem s_oscillator.html\#more. 